
zoratrance
Gesin:
MG
CENTRO DE ESTUDIOS
INTERDISCIPLINARIOS SOBRE MUJERES
MAESTRIA "PODER Y SOCIEDAD
DESDE LA PROBLEMATICA DE GÉNERO"

\title{
El hostigamiento sexual hacia mujeres afrocostarricenses como manifestación racista y misógina en espacios laborales
}

\author{
Dannia Gamboa Solís * \\ Verónica González Prado* * \\ Sigrid Solano Moraga* * *
}

\section{Resumen}

Esta investigación evalúa el hostigamiento sexual hacia las mujeres afrocostarricenses en el ámbito laboral. El objetivo primordial se centra en estudiar las manifestaciones y efectos del hostigamiento sexual laboral en mujeres afrocostarricenses para la inclusión de una mirada interseccional en la prevención, sanción y erradicación de estas formas de violencia que afectan sus derechos humanos. Este estudio surge en la coyuntura de la emergencia por Covid 19, por lo que se emplearon herramientas de conexión remota para obtener resultados provenientes de treinta y tres mujeres de diferentes zonas de Costa Rica. Por medio de las respuestas de estas encuestadas se exponen los resultados sobre las

* Periodista con formación en perspectiva de género en los derechos humanos. Contacto: danniagamboa10@gmail.com

* * Licenciada en psicología con formación en perspectiva de género en los derechos humanos. Experiencia en derechos sexuales y reproductivos y en la prevención y atención de la violencia contra las mujeres. Contacto: veroprado9220@gmail.com

* * * Filóloga y docente universitaria con formación de género en los derechos humanos. Contacto: sisomo20@gmail.com

Gamboa Solís, Dannia; González Prado, Verónica; Solano Moraga, Sigrid. "El hostigamiento sexual hacia mujeres afrocostarricenses como manifestación racista y misógina en espacios laborales" en Zona Franca. Revista del Centro de estudios Interdisciplinario sobre las Mujeres, y de la Maestría poder y sociedad desde la problemática de Género, №29, 2021 pp. 288-323. ISSN, 2545-6504 Recibido: 12 de mayo 2021; Aceptado: 08 de noviembre 2021.

Revista Zona Franca- Centro de estudios interdisciplinario sobre las mujeres (CEIM)- Maestría poder y sociedad desde la problemática de género (MG), Rosario, Argentina. ISSN, 2545-6504 http://zonafranca.unr.edu.ar/index.php/ZonaFrancal Número 29 (2021). 
manifestaciones del hostigamiento sexual, las percepciones, la identificación del problema y los efectos sobre sus víctimas.

Palabras clave: racismo, misoginia, hostigamiento sexual, interseccionalidad, mujeres afrocostarricenses.

\section{Sexual harassment in workplaces perpetrated to Afro-Costa Rican women}

\section{Abstract}

This research studies sexual harassment in workplaces perpetrated to AfroCosta Rican women. The study focuses on the demonstrations and effects of sexual harassment at work suffered by afro-Costa Rican women, according to an intersectional perspective on the prevention, punishment, and eradication of these forms of violence that affect their human rights. This study initiates during Covid 19 emergency and presents the main results obtained by the responses of thirty- three women, as well as the results on the manifestations of sexual harassment, perceptions, identification of the problem, and the effects on its victims. According to the conclusions, sexual harassment must be evaluated from different circumstances that cross female realities.

Keywords: racism, misogyny, sexual harassment, intersectionality, AfroCosta Rican women.

La violencia contra las mujeres refiere a las acciones o conductas que suceden en todos los espacios de participación social, política, económica y cultural. El reconocimiento de ciertas formas de violencia puede considerarse casi nulo, incluso por parte de quienes las sufren. Entre estas manifestaciones se ubica el hostigamiento sexual pues ocurre de manera sutil, con lo que se torna difícil de aprehender, pues su ejercicio simbólico ha estado impregnado en el imaginario colectivo.

De acuerdo con Sagot (2008), la discriminación y la violencia contra las mujeres se confirma como "un componente estructural del sistema de opresión de género" (p. 36), el cual se gesta por medio de relaciones de poder. Por lo tanto,

Revista Zona Franca- Centro de estudios interdisciplinario sobre las mujeres (CEIM)- Maestría poder y sociedad desde la problemática de género (MG), Rosario, Argentina. ISSN, 2545-6504 http://zonafranca.unr.edu.ar/index.php/ZonaFranca| Número 29 (2021). 
aspectos como la edad, el género, la raza, la condición socioeconómica y las posiciones de jerarquía sostienen estas conductas y las legitiman.

En el caso específico de las mujeres negras, esta forma de opresión se vive a raíz de dos componentes que, aunque diferentes, son interdependientes: el género y la etnia; ambos se viven como experiencias distintas, pero de forma paralela en el mismo cuerpo y atentan contra el derecho a tener una vida libre de violencia.

Los discursos socialmente construidos que racializan los cuerpos de las mujeres negras y los reducen a objetos sexualizados exponen manifestaciones del hostigamiento sexual relacionadas con miradas lascivas y ciertas expresiones misóginas -dadas principalmente por hombres-dirigidas a partes específicas de sus cuerpos, entre ellas, las caderas, las piernas, el cabello y el color de piel.

En Costa Rica, de acuerdo con el Inamu (2016), fue hasta la década de los 90 que se adoptó la ley $N^{\circ} 7476$, la cual sanciona el hostigamiento sexual en los ámbitos del empleo y la docencia. Según datos estadísticos de la Defensoría de los Habitantes, el $97 \%$ de los casos que se tramitan por acoso u hostigamiento sexual en el país corresponde a denuncias interpuestas por mujeres, porcentaje que desvela una problemática relacionada con la violencia que se ejerce mayoritariamente contra las mujeres debido a su género y que constituye una barrera para su desempeño profesional.

Por su parte, existen pocos estudios sobre el caso del hostigamiento sexual contra las mujeres negras en el país, aun cuando la población que se autoidentifica como afrodescendiente es del 7,77\% de un total de 4600000 de habitantes; porcentaje del cual 161.402 son mujeres, de acuerdo con el Inamu, basado en el censo nacional de 2011.

Según otros datos del mismo censo en relación con esta población, se indica que el porcentaje con algún tipo de trabajo remunerado es del 54,4\%. De este porcentaje solamente el $35,6 \%$ de las mujeres afrodescendientes tiene esta

Revista Zona Franca- Centro de estudios interdisciplinario sobre las mujeres (CEIM)- Maestría poder y sociedad desde la problemática de género (MG), Rosario, Argentina. ISSN, 2545-6504 http://zonafranca.unr.edu.ar/index.php/ZonaFranca| Número 29 (2021). 
condición en comparación con el $72 \%$ de los hombres afrodescendientes. Como se observa, para esta población de mujeres afrodescendientes persisten las brechas raciales.

Ante estos datos, esta investigación pretende visibilizar la intersección de distintas formas de opresión contra las mujeres, en la que la vivencia del racismo y la misoginia se experimentan y se desarrollan en un mismo cuerpo. En el caso del hostigamiento sexual laboral, se muestra como expresión implícita cuya finalidad es la expulsión de las mujeres de los espacios públicos, históricamente conservados para los hombres.

Por lo tanto, a partir de la interseccionalidad presente en los casos de violencia sexual -específicamente en el hostigamiento - que viven las mujeres a diario, este artículo tiene como objetivo estudiar las manifestaciones y efectos del hostigamiento sexual laboral en mujeres afrocostarricenses con la finalidad de incluir una perspectiva interseccional con la que se prevengan, sancionen y erradiquen estas formas de violencia. A continuación, se desglosan tres fines específicos del estudio:

- Identificar las principales manifestaciones de hostigamiento sexual que han sufrido mujeres afrocostarricenses en sus ámbitos laborales.

- Exponer los efectos de esta forma de violencia en la vida de las mujeres afrocostarricenses desde una perspectiva de género en los derechos humanos.

- Incorporar una mirada interseccional para el entendimiento de una problemática compleja, tal como lo es el hostigamiento sexual laboral.

Con el fin de desarrollar estos objetivos, se propone una metodología enfocada en una perspectiva interseccional sobre la violencia y la discriminación hacia las mujeres; se emplea, por lo tanto, el género, el racismo y los derechos humanos de las mujeres como ejes fundamentales. Aunado a esto, se emplea un enfoque cualitativo que se caracteriza por mantener una lógica inductiva con la que se 
establece una relación dialéctica entre teoría y la percepción, así como la vivencia de las personas.

Esta investigación propone, a su vez, profundizar en la subjetividad de la población que participa en el proceso, para lo que se toma en cuenta a Hernández, Fernández y Baptista (2010), en relación con sus estipulados sobre la investigación cualitativa, en donde la perspectiva y la experiencia de las personas es fundamental y parte de esta para la construcción de conocimiento.

Asimismo, el alcance de la investigación es de tipo descriptiva, en este sentido, la especificidad del estudio se centrará en la realidad de las mujeres afrocostarricenses en relación con las manifestaciones y consecuencias del hostigamiento sexual dentro del contexto laboral.

\section{Muestra}

Se cuenta con una muestra seleccionada a conveniencia y no representativa, ya que se trata de un estudio cualitativo que busca generar análisis a partir de las percepciones de las participantes. Esta muestra se conforma por treinta y tres mujeres que se identifican como afrocostarricenses y que se caracterizan por estar activas laboralmente (al momento de realizar la investigación), residir en diferentes sectores del país -predominantemente en las zonas de Limón y Siquirres- y tener un promedio de edad de 39 años.

\section{Recolección de datos}

Se recolectaron datos para su posterior análisis, para lo que se empleó un cuestionario compuesto por dieciséis preguntas, de las cuales las dos primeras se centran en identificar características sociodemográficas. El resto de las preguntas se orienta a la identificación de los conocimientos sobre hostigamiento sexual, la asociación entre esta forma de violencia y el racismo, así como la vivencia de

Revista Zona Franca- Centro de estudios interdisciplinario sobre las mujeres (CEIM)- Maestría poder y sociedad desde la problemática de género (MG), Rosario, Argentina. ISSN, 2545-6504 http://zonafranca.unr.edu.ar/index.php/ZonaFrancal Número 29 (2021). 
situaciones de acoso en sus espacios de trabajo y, finalmente, los procesos de denuncia que se han seguido en casos de hostigamiento sexual laboral.

\section{Procedimiento}

El cuestionario fue respondido a través de la plataforma digital Formularios de Google, modalidad empleada debido al contexto de la pandemia por COVID-19. La información recopilada fue debidamente sistematizada por medio de matrices de texto y gráficos, para proceder con el respectivo análisis.

\section{Conceptos necesarios para la comprensión del estudio}

\section{Racismo, discriminación e interseccionalidad}

Dado que los conceptos de raza y etnia se tienden a confundir, nos basamos en los estipulados de Hopenhayn y Bello para diferenciarlos; los autores proponen lo siguiente: "Mientras la raza se asocia a distinciones biológicas atribuidas a genotipos y fenotipos, especialmente con relación al color de la piel, la etnicidad se vincula a factores de orden cultural, si bien con frecuencia ambas categorías son difícilmente separables" (2001, p. 7.).

Entendemos, con la cita, que las supuestas diferencias biológicas sustentadas en estudios cientificistas son la base encontrada por los discursos de poder para la clasificación humana, desde diferentes períodos históricos, como inferiores a partir de la concepción de las "razas". Con ello, se ejerce, además, la "negación del otro" como medio de exclusión, e implica para esos "otros" el no tener posibilidades de acceder a diferentes dinámicas sociales y económicas. De esta manera, los cuerpos "no blancos" quedan supeditados a los cuerpos con poder, lo que origina el racismo, explicado, para el contexto americano del siguiente modo:

El racismo regula las clasificaciones de comunidades humanas en base a

la sangre y al color de piel. Mientras que en España, "conversos" y

Revista Zona Franca- Centro de estudios interdisciplinario sobre las mujeres (CEIM)- Maestría poder y sociedad desde la problemática de género (MG), Rosario, Argentina. ISSN, 2545-6504 http://zonafranca.unr.edu.ar/index.php/ZonaFrancal Número 29 (2021).

Página 293 
"moriscos" marcaban la mezcla de sangre con la religión, en América, fueron los/las mulatos/mulatas y los/las mestizas quienes ocuparon el lugar equivalente. Quienes clasificaban, quienes controlaban el saber, eran hombres cristianos y blancos (Mignolo 2000: 9).

Las estructuras vejatorias impuestas por cuestiones de piel se ligan, en este estudio, a las de género; se debe considerar que las mujeres negras reciben una doble discriminación; las mujeres por categorizarse como tales sufren de desigualdades por el establecimiento de jerarquías para mantener un supuesto orden social. Estas jerarquías proponen la supremacía de un género (masculino) sobre el otro (femenino) sustentado en el patriarcado, el cual se entiende como:

el poder de los padres: un sistema familiar y social, ideológico y político con el que los hombres - a través de la fuerza, la presión directa, los rituales, la tradición, la ley o el lenguaje, las costumbres, la etiqueta, la educación y la división del trabajo- determinan cuál es o no es el papel que las mujeres deben interpretar con el fin de estar en toda circunstancia sometidas al varón (Rich, citado por Carvajal y Delvó 2010: 61).

Lugones (2012) propone que, en América, desde la época colonial, las mujeres racializadas (negras o indígenas) perdían su condición humana, pues además de ejercer trabajos pesados, eran explotadas sexualmente por su percepción como objetos a la orden de los hombres colonizadores:

Las hembras no-blancas eran consideradas animales en el sentido profundo de ser "sin género", marcadas sexualmente como hembras pero sin las características de la femineidad. Las hembras racializadas como seres inferiores pasaron de ser concebidas como animales a ser concebidas como símiles de mujer en tanto versiones de "mujer" como fueron necesarias para los procesos del capitalismo eurocentrado global (Lugones 2012: 45).

Revista Zona Franca- Centro de estudios interdisciplinario sobre las mujeres (CEIM)- Maestría poder y sociedad desde la problemática de género (MG), Rosario, Argentina. ISSN, 2545-6504 http://zonafranca.unr.edu.ar/index.php/ZonaFrancal Número 29 (2021). 
Se entiende, entonces, que las mujeres no blancas sufren de un estatus más bajo que la mujer de por sí discriminada, al mismo tiempo que pierden sus cualidades femeninas para ser objetos de trabajo. Estas ideas, a pesar de la ausencia de razones científicas, es lo que podríamos denominar la base de una sociedad que define jerárquicamente unos cuerpos sobre otros. De este modo, para el caso de Latinoamérica, la imposición hegemónica de un modelo universal de mujer es mecanismo que buscar moldear las experiencias femeninas desde contextos de dominación históricos (Stolcke en Wade, Urrea Giraldo y Vigoya, 2008: 16).

Como se observa previamente, los enfoques de estudio sobre las problemáticas que experimentan las mujeres se deben vislumbrar desde las diferentes aristas que logren capturar sus realidades y discursos de poder. El término fue propuesto por Kimberly Crenshaw, en 1989 para contrarrestar la tendencia de visualizar el género y la raza por separado. De este modo, Crewshaw analiza el término:

The process of social construction or categorization is not a unilateral process: there is a give and take in any system of categorization. One need only think of the category "black" to understand that it is not a one-way street. There is, of course, unequal power, but there's nonetheless some degree of agency that people can and do exert in the politics of naming (Crenshaw 1995: s.p.).

La autora propone que existe un sistema de categorización social que no permite más que una arista en su entendimiento. De este modo, por ejemplo, la problemática de una persona negra se estudiaba en sí misma, sin asociarla con otras categorías como clase, casta, raza, color, etnia, religión, origen, orientación sexual, etc.

Revista Zona Franca- Centro de estudios interdisciplinario sobre las mujeres (CEIM)- Maestría poder y sociedad desde la problemática de género (MG), Rosario, Argentina. ISSN, 2545-6504 http://zonafranca.unr.edu.ar/index.php/ZonaFrancal Número 29 (2021). 
Este enfoque es indispensable para comprender que la problemática del hostigamiento sexual tiene matices significativos si es sufrido por una mujer negra, quien es construida y "marcada" por su color de piel. De manera que, para el caso de las mujeres racializadas, es imposible separar su experiencia de las opresiones raciales, clasistas y sexuales, como lo plantean los feminismos negros (Wade en Wade, Urrea Giraldo y Vigoya, 2008: p. 20).

\section{El cuerpo femenino y negro}

Con la finalidad de entender los comportamientos discriminatorios que viven las mujeres afrocostarricenses, es necesario explicar cómo se concibe simbólicamente el cuerpo de estas en el ideario fundado en la cultura patriarcal desde la colonia, tal y como se fundamentó en el apartado previo.

Bourdieu (1998) explica la relación del poder simbólico con el cuerpo: "La fuerza simbólica es una forma de poder que se ejerce directamente sobre los cuerpos y como por arte de magia, al margen de cualquier coacción física" (54). De este modo, las mujeres se someten involuntariamente a las conductas subordinantes, propias de los discursos ideologizantes presentes en la cultura.

De este modo, la dominación sobre los cuerpos se naturaliza y se pone en práctica tanto por los dominados como por los dominadores, quienes la admiten inconscientemente, con lo que se socializa o se universaliza:

La violencia simbólica se instituye a través de la adhesión que el dominado se siente obligado a conceder al dominador (por consiguiente, a la dominación) cuando no dispone, para imaginarla o para imaginarse a sí mismo o, mejor dicho, para imaginar la relación que tiene con él, de otro instrumento de conocimiento que aquel que comparte con el dominador y que, al no ser más que la forma asimilada de la relación de dominación, hacen que esa relación parezca natural; o, en otras palabras, cuando los esquemas que pone en práctica para percibirse y apreciarse, o para percibir y apreciar a los dominadores (alto/bajo, masculino/femenino,

Revista Zona Franca- Centro de estudios interdisciplinario sobre las mujeres (CEIM)- Maestría poder y sociedad desde la problemática de género (MG), Rosario, Argentina. ISSN, 2545-6504 http://zonafranca.unr.edu.ar/index.php/ZonaFrancal Número 29 (2021). 
blanco/negro, etc.), son el producto de la asimilación de las clasificaciones, de ese modo naturalizadas, de las que su ser social es el producto (Bourdieu 1998: 52).

Esta proyección de los cuerpos como parte de una naturaleza determinada se consolida a partir de estructuras fundamentales, que se simplifican a partir de dualidades: blanco/negro, delgado/ grueso, bueno/ malo, lindo/ feo, que son conocidas y reconocidas socialmente: "el cuerpo percibido está doblemente determinado desde un punto de vista social" (Bourdieu 1998: 85). En ese reconocimiento se basa la efectividad de ese poder simbólico, que establece la contraposición entre un cuerpo real contra uno ideal o entre patrones aceptados o no aceptados.

A estas valoraciones construidas socialmente, se agrega que son históricas y, además, permiten "asociar unas propiedades «psicológicas» y «morales» a unos rasgos corporales o fisonómicos. Esta idea propone la existencia de estereotipos que calan en el ideario cultural sin deconstrucción, en muchos casos.

De esta manera, la generación de valores y percepciones subjetivas, aunado a un pasado colonial, expone sobre los cuerpos de las mujeres negras e indígenas preconcepciones sobre sus cuerpos y su forma de ser, en general, que desde un vistazo al pasado han contrastado con las de las mujeres blancas:

Históricamente, la caracterización de las mujeres europeas blancas como sexualmente pasivas y física y mentalmente frágiles las colocó en oposición a las mujeres colonizadas, blancas, incluidas las mujeres esclavas, quienes, en cambio, fueron caracterizadas a lo largo de una gama de perversión y agresiones sexuales y, también, consideradas lo suficientemente fuertes como para cambiar cualquier tipo de trabajo (Lugones 2012: 49).

Revista Zona Franca- Centro de estudios interdisciplinario sobre las mujeres (CEIM)- Maestría poder y sociedad desde la problemática de género (MG), Rosario, Argentina. ISSN, 2545-6504 http://zonafranca.unr.edu.ar/index.php/ZonaFrancal Número 29 (2021). 
Estas cargas simbólicas sobre los cuerpos de las mujeres negras establecidos en un pasado cuentan con resabios en la contemporaneidad, que evaluaremos en la presente investigación.

Finalmente, otro modo de entender la sexualización de los cuerpos de las mujeres afrodescendientes tiene que ver con el deseo ambivalente que siente el sujeto hacia "el otro". En este caso, Wade (basado en Fanon, Butler, Stoller y el psicoanálisis, entre otros) indica que, históricamente, el otro (en este caso, la mujer afrodescendiente), es amenazante pero a la vez deseado: "El otro, que es el inconsciente y también la madre perdida, es el objeto del deseo, pero al mismo tiempo, objeto de agresión porque está prohibido y representa la ruptura de la unidad" (Wade, 2008, p. 59).

\section{Hostigamiento sexual}

La Ley contra el hostigamiento sexual en el empleo y la docencia en Costa Rica entiende por hostigamiento sexual o acoso sexual: "toda conducta sexual indeseada por quien la recibe, reiterada y que provoque efectos perjudiciales en los siguientes casos: a) Condiciones materiales de empleo y docencia, b) Desempeño y cumplimiento laboral o educativo, c) Estado general de bienestar personal" (2011, art. 3).

El hostigamiento, de acuerdo con Carvajal y Delvó (2010), pasa desapercibido o, lo que es similar, se naturaliza, lo anterior porque parece que las mujeres lo "pasan como algo que tiene que suceder entre el jefe y la subalterna, el docente y la/el estudiante, entre compañeras y compañeros de trabajo o de estudio" (Carvajal y Delvó 2010: 62). Esto se debe a conductas que son atribuidas a los hombres; de manera que, desde el discurso de las masculinidades, él debe conquistar y ella ser un receptáculo del deseo de él.

\section{Manifestaciones del hostigamiento sexual}

Revista Zona Franca- Centro de estudios interdisciplinario sobre las mujeres (CEIM)- Maestría poder y sociedad desde la problemática de género (MG), Rosario, Argentina. ISSN, 2545-6504 http://zonafranca.unr.edu.ar/index.php/ZonaFranca| Número 29 (2021). 
El Tercer Estado de los Derechos Humanos de las Mujeres (Inamu, 2019) manifiesta que esta conducta es frecuente en el país y se basa, para comprobarlo, en la Encuesta Nacional sobre Salud Sexual y Reproductiva de 2015, que exterioriza que las mujeres sufren en mayor medida de conductas que recaen en la definición de hostigamiento sexual: "Entre las más comunes se encuentran "expresiones o piropos" ( $73 \%$ de las mujeres), "miradas insinuantes" (65\% de las mujeres), "chistes sexistas" ( $57 \%$ de las mujeres) y "comentarios sobre el cuerpo o manera de vestir" (56\% de las mujeres) (Inamu 2017: 214).

De acuerdo con la Organización Internacional del Trabajo (OIT) y Reyes (2008), el hostigamiento puede presentarse de dos maneras:

a. Por chantaje: cuando se condiciona a la víctima a que acceda a comportamientos de connotación sexual, con la promesa de algún beneficio, como aumento de sueldo, promoción laboral, etc. Este es el denominado quid quo pro ("tome y deme").

b. Con un ambiente hostil de trabajo: intimida o humilla a la víctima.

La OIT (2013), además, expone que los comportamientos que se califican como acoso sexual pueden ser de naturaleza:

a. Física: violencia física, tocamientos, acercamientos innecesarios.

b. Verbal: comentarios y preguntas sobre el aspecto, el estilo de vida, la orientación sexual, llamadas de teléfono ofensivas.

c. No verbal: silbidos, gestos de connotación sexual, presentación de objetos pornográficos (213: 2).

A estas manifestaciones, Ramírez (2016) agrega una cuarta y es la digital, la cual incluye mensajes escritos con contenido sexual, dirigidos al correo electrónico 
o por mensajería de texto que puede presentarse como videos, post, gifs, audios dedicados a la persona hostigada o tomar fotografías sin consentimiento.

Asimismo, se presentan algunos ejemplos de hostigamiento sexual:

Tabla 1. Ejemplos de conductas que responden a hostigamiento sexual (2013):

- Contacto

físico

innecesario y no deseado.

- Observaciones molestas y otras formas de acoso verbal.

- Miradas lascivas y gestos relacionados con la sexualidad.

- Petición de favores sexuales.

- Insultos, observaciones, bromas e insinuaciones de carácter sexual.

- Comentarios, bromas, gestos o miradas sexuales.

- Manoseos, jalones o pellizcos en forma sexual.

- Restregar a la víctima contra alguien de un modo sexual.

- Propagar rumores sexuales acerca de la víctima.
- La utilización o exhibición de material pornográfico.

- Jalar la ropa de manera sexual.

- Mostrar, dar o dejar imágenes sexuales, fotografías, ilustraciones, mensajes o notas sexuales.

- Escritos, mensajes (pintas, grafitis) sexuales acerca de la víctima, en paredes de los baños, vestuarios, etc.

- Forzar a besar a alguien o a algo más que besar

- Llamar a la víctima "gay" o "lesbiana".

- Espiar mientras se cambia o está encerrada en un sanitario.

- La utilización o exhibición de material pornográfico.

\section{Consecuencias del hostigamiento sexual}

Revista Zona Franca- Centro de estudios interdisciplinario sobre las mujeres (CEIM)- Maestría poder y sociedad desde la problemática de género (MG), Rosario, Argentina. ISSN, 2545-6504 http://zonafranca.unr.edu.ar/index.php/ZonaFranca| Número 29 (2021). 
De acuerdo con Carvajal y Delvó (2010), los efectos del hostigamiento sexual son inconmensurables y perduran en el tiempo. De este modo, el hostigamiento sexual influye en el desempeño laboral e impacta la salud de las personas y afecta a las víctimas, a los empleadores o jefes de la institución y a la sociedad en general.

Las víctimas pueden sufrir enfermedades físicas o mentales, tener cambios de comportamiento o sufrimiento psicológico, e incluso, sufrir un aumento de accidentalidad. Los segundos, pueden verse afectados en la productividad de la empresa o de la institución, en la rotación de recursos humanos, en gastos en indemnizaciones y en el deterioro de las relaciones entre personas trabajadoras. Respecto de la sociedad, esta se ve afectada en los costes por el reingreso de víctimas, el incremento de violencia y segregación laborales, dificultad en el acceso de mujeres a trabajos tradicionalmente dominados por hombres.

Por otro lado, en el ámbito laboral, el hostigamiento corresponde para las víctimas mujeres en un aspecto negativo que impide su crecimiento como trabajadoras:

Si bien la tasa de participación de las mujeres en el mercado laboral se ha incrementado, esto no implica necesariamente que el ámbito laboral esté evolucionando de manera positiva para las mujeres. Aun nos encontramos con procesos de discriminación que se traducen en obstáculos para el desarrollo de la trayectoria profesional y laboral de las mujeres en igualdad de condiciones que los hombres (Ayala, Cabezas y Filippis 2011: 4).

Con los datos anteriores, consideramos que es indispensable evaluar el hostigamiento como conducta naturalizada y su intersección con el eje de razaetnia en la cultura costarricense, con el fin de brindar datos respecto de la percepción del acoso por las mismas mujeres que lo han sufrido.

\section{Instrumento}

Revista Zona Franca- Centro de estudios interdisciplinario sobre las mujeres (CEIM)- Maestría poder y sociedad desde la problemática de género (MG), Rosario, Argentina. ISSN, 2545-6504 http://zonafranca.unr.edu.ar/index.php/ZonaFranca| Número 29 (2021). 
Ante la coyuntura mundial sucedida por la emergencia SARS-CoV-2, acontecida desde el 2020, la investigación se realizó de modo virtual, por medio de un consentimiento informado y anónimo, entre los días 22 al 27 de octubre de ese año. Para proceder a un acercamiento de las actitudes, percepciones y conocimientos sobre hostigamiento sexual laboral por parte de las mujeres que se identificaron como afrocostarricenses, se llevó a cabo un cuestionario con un total de 14 preguntas y se difundió a través de la plataforma Google Forms (Formularios de Google). Por estas circunstancias de virtualidad, y con la motivación de tener un panorama general y no disminuir el punto de alcance, no se eligió una zona del país en particular.

La encuesta contempló distintos ejes de interés relacionados con el hostigamiento sexual laboral, entre los que se destacan:

$\rightarrow$ Conocimiento general sobre hostigamiento sexual.

$\rightarrow$ Percepción del hostigamiento sexual en sus lugares de trabajo.

$\rightarrow$ Frecuencia con la cual ocurre.

$\rightarrow$ Sexo de las personas hostigadoras.

$\rightarrow$ Relación laboral con la persona hostigadora.

$\rightarrow$ Relación y percepción entre hostigamiento sexual y discriminación racial.

$\rightarrow$ Principales manifestaciones del hostigamiento sexual.

$\rightarrow$ Denuncias y sus resoluciones.

\section{Sistematización y análisis de la información}

El proceso de sistematizar y analizar los datos obtenidos en el cuestionario partió de la organización de la información a través de gráficos de texto.

Para ello, a partir del análisis de cada una de las respuestas, se desprendieron tres categorías:

Revista Zona Franca- Centro de estudios interdisciplinario sobre las mujeres (CEIM)- Maestría poder y sociedad desde la problemática de género (MG), Rosario, Argentina. ISSN, 2545-6504 http://zonafranca.unr.edu.ar/index.php/ZonaFranca| Número 29 (2021). 
1. Racialización del hostigamiento sexual: relación entre hostigamiento sexual y hostigamiento racial.

2. Manifestaciones: identificación y percepciones del hostigamiento sexual en el ámbito laboral.

3. Efectos: consecuencias del hostigamiento sexual en el trabajo, la salud y la afectación de este en los derechos humanos de las mujeres.

Estas categorías permitieron un proceso de integración y reflexión junto con la teoría previamente identificada, así como la generación de insumos para próximas investigaciones en la materia.

\section{Análisis de resultados}

Por medio de la encuesta, se recopiló la información de treinta y tres mujeres residentes de diferentes partes del país (Figura 1), las cuales oscilan en un promedio de treinta y siete años.

\section{Figura 1. Lugar de residencia}

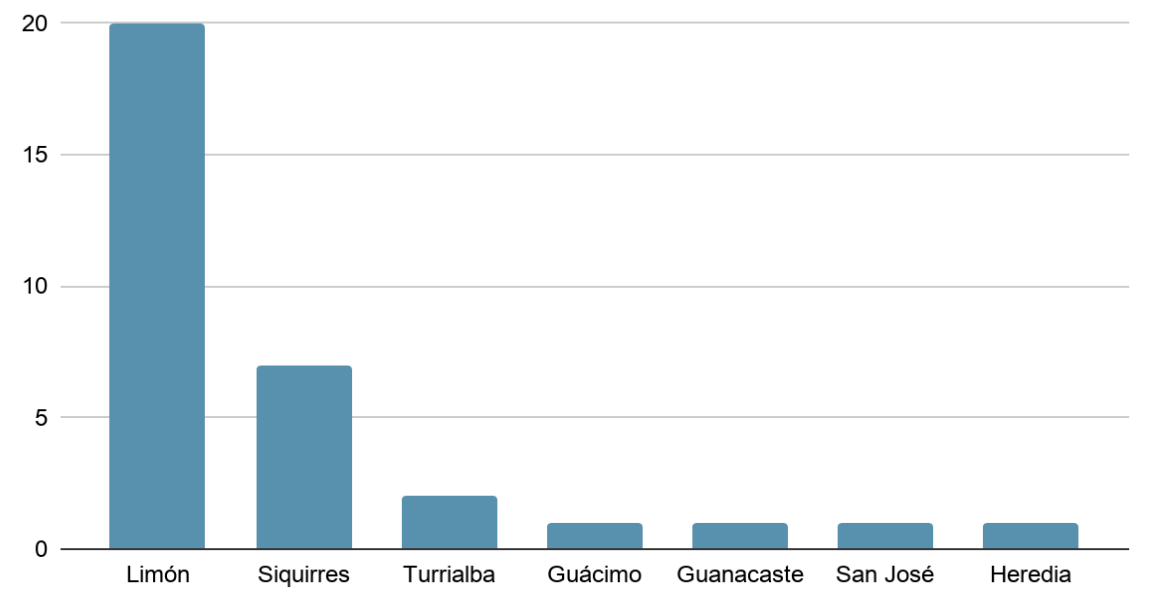

Nota: Elaboración propia. 
Sobre este resultado, es interesante ver que la mayoría de entrevistadas proviene de la provincia de (Limón y Siquirres), ya que coincide con el espacio en el que tradicionalmente se ubica la población del país. De acuerdo con el Inamu, en el cantón Central de Limón y de Talamanca vive un $45 \%$ de la población que se identifica como afrodescendiente.

\section{La racialización del hostigamiento sexual}

De acuerdo con las respuestas recopiladas, el 100\% de las mujeres participantes ha escuchado hablar sobre hostigamiento sexual. No obstante, en el transcurso del cuestionario se evidencian incongruencias en sus respuestas con respecto a su identificación, así como de la correlación entre hostigamiento sexual y hostigamiento racial.

De acuerdo con Buchanan y Fitzgerald (2018), ambas formas de hostigamiento se entrelazan en sus manifestaciones y en conjunto posicionan a las mujeres afrodescendientes en un espacio de mayor vulnerabilidad frente a estas manifestaciones de violencia dentro de sus espacios laborales.

La atención del hostigamiento sexual en mujeres afrodescendientes corresponde a una observación que trasciende el género y que a la vez complejiza las relaciones sociales, lo cual también se vuelve aún más profundo para quienes lo experimentan debido a una naturalización de la violencia de la cual son víctimas.

Por ello, los aportes de María Lugones sobre el análisis interseccional son valiosos para este trabajo. Lugones (2005) establece que las diferentes formas de opresión no funcionan en una capacidad de divisibles; es decir, la raza y el género se manifiestan en una fusión que no es separable, por lo que afirmar que la interseccionalidad es solamente un entrecruce de opresiones que se pueden separar, es solapar o encubrir esta inseparabilidad. Lo que provoca que a "dondequiera que miremos, encontramos el solapamiento de opresiones que nos incapacita para percibir y resistir a las opresiones como mezcladas y fundidas" (70).

Revista Zona Franca- Centro de estudios interdisciplinario sobre las mujeres (CEIM)- Maestría poder y sociedad desde la problemática de género (MG), Rosario, Argentina. ISSN, 2545-6504 http://zonafranca.unr.edu.ar/index.php/ZonaFranca| Número 29 (2021). 
Este solapamiento simbólico de las opresiones se observa a través del cuestionario aplicado, partiendo por la pregunta sobre considerar que existe una relación entre el hostigamiento sexual y la etnia. En este caso, el $58 \%$ de las respuestas indicaron que no existe dicha relación, mientras que el $42 \%$ afirma un entrelace.

Las respuestas previas varían en relación con la pregunta sobre haber recibido comentarios o bromas sexuales con respecto de su cuerpo, con la que un $79 \%$ de las respuestas confirman haber sido víctimas de esta forma de hostigamiento $\mathrm{y}$, entre los tipos de comentarios e insinuaciones que han presenciado, prevalecen los relacionados con su color de piel y partes específicas de su cuerpo que han sido comúnmente hipersexualizadas en las mujeres negras (Figura 2).

De acuerdo con Bourdieu (1998), la dominación que se añade sobre los cuerpos se tiende a naturalizar y, por ende, se universaliza. A partir de procesos de socialización sumergidos en lógicas patriarcales, tampoco se cuestiona la dominación masculina que ha prevalecido sobre los cuerpos de las mujeres. Foucault, por su parte, expone sobre la exploración del cuerpo como territorio de inscripción de las relaciones de poder:

el cuerpo es el territorio donde se inscribe la cultura, la clase social, la etnicidad, el modo de estar y, sobre todo, el género. Dentro de estos constructos, el género y la sexualidad, expresados en el cuerpo como lo visible, atribuyen sentidos a las prácticas individuales y sociales que son reconocidas y al mismo tiempo reproducidas por los sujetos (1992: 166).

Por ello, la arbitrariedad cultural de ciertos estereotipos pareciera no encontrar la necesidad de ser deconstruidos en la contemporaneidad; están tan impregnados en los procesos de socialización que llegan a carecer de sentido y reconocimiento por parte de las mismas personas que sufren la opresión.

Revista Zona Franca- Centro de estudios interdisciplinario sobre las mujeres (CEIM)- Maestría poder y sociedad desde la problemática de género (MG), Rosario, Argentina. ISSN, 2545-6504 http://zonafranca.unr.edu.ar/index.php/ZonaFrancal Número 29 (2021). 
A lo anterior se suma a la exaltación impuesta sobre la sexualidad de las mujeres negras, aunado en la caracterización histórica de las mujeres esclavas sobre los elementos de la perversión y las agresiones sexuales, como se explicó previamente.

\section{Figura 2. Comentarios que prevalecen en el hostigamiento sexual contra mujeres afrocostarricenses}

15

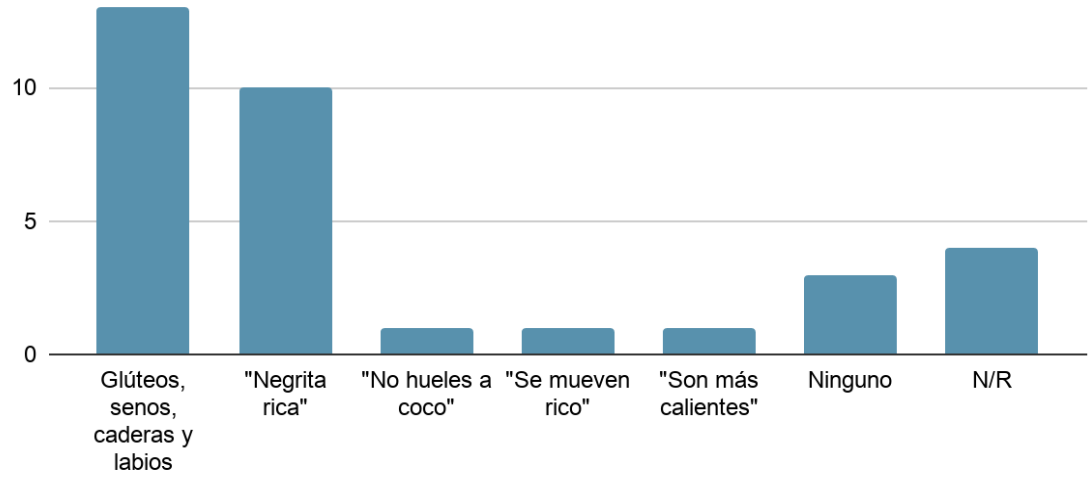

Nota: Elaboración propia.

La fusión de ambos sistemas de opresión, patriarcado y racismo, se arrastra hasta la actualidad, dado que las percepciones de las mujeres entrevistadas evidencian la inseparabilidad entre hostigamientos sexual y racial, en el momento de expresar los (mal) nombrados "elogios" o comentarios con respecto del cuerpo de las mujeres afrocostarricenses. Además, se sostiene un carácter permisivo por parte de los hostigadores a través de una hipersexualización de ellas.

Con base en lo anterior, la interpretación correlacional entre ambas formas de hostigamiento se torna divergente en el caso de si las entrevistadas consideran que el trato recibido como mujeres es distinto al que se les brinda a las mujeres no racializadas. Un 58\% afirma que efectivamente hay una diferencia. Es importante resaltar que, en este ítem en particular, la pregunta no se relaciona directamente 
con el hostigamiento sexual, por lo que da paso a una visión que se aleja de un reconocimiento de la violencia sexual desde la categoría etnia.

Por lo tanto, los comentarios en relación con la diferenciación afirmativa entre mujeres afrocostarricenses y mujeres no racializadas se centran en una caracterización histórica de subordinación de las personas negras que ha resultado más expuesta a través de los años y que no se ubica explícitamente en una connotación sexual del cuerpo, pero que no deja de ser hostigamiento racial:

- Cargas laborales diferenciadas, específicamente entre posiciones de jefatura, donde se minimiza a la persona y se subestiman sus capacidades para desempeñarse laboralmente.

- Invisibilización de las personas afrodescendientes en los medios de comunicación y la publicidad.

- Discriminación o trato diferenciado en lugares públicos. Por ejemplo: al hacer reservaciones en un restaurante, por lo general les otorgan espacios en lugares poco visibles.

- Discriminación por la forma de hablar y por el peso.

- No ser tomadas en cuenta.

El color de piel, de acuerdo con las respuestas, marca el trato diferenciado al señalar que se es "negra", como lo indica una de las respuestas. Y continúa explicando que no es común que las personas se dirijan a una no racializada como "blanca" o: "a mí no me gustan las negras porque se ven como tierrosas". La sociedad sigue siendo muy discriminatoria en estos aspectos (mujer, 25 años).

A partir de distintos procesos históricos se ha aceptado una competición por la supervivencia entre las "razas humanas" y, en esta lucha, también se justifica desde teorías como la darwiniana- el proceso moderno de colonización y la construcción categórica de "los otros" (Sánchez-Arteaga, Sepúlveda y El-Hani, 2013: 61).

Revista Zona Franca- Centro de estudios interdisciplinario sobre las mujeres (CEIM)- Maestría poder y sociedad desde la problemática de género (MG), Rosario, Argentina. ISSN, 2545-6504 http://zonafranca.unr.edu.ar/index.php/ZonaFranca| Número 29 (2021). 
Dentro de un sistema global de poder, mediante el cual se apoyan y sustentan las interseccionalidades, se establecen las zonas del ser y no ser, una zona implantada no por cuestiones geográficas, sino por una posición de las relaciones de poder. Mediante esta línea divisoria, la raza se ve atravesada por las relaciones de opresión que también involucran clase, sexualidad, género, etnia, entre muchas, como una forma de jerarquización de cuerpos que importan más que otros. Hopenhayn y Bello (2001) expresan que:

\begin{abstract}
el peso del argumento racial ha pasado del discurso científico al imaginario social, sobre la base de variaciones fenotípicas con que cada sociedad construye significados en el contexto de sus experiencias históricas [...] Esta negación del otro se expresa de distintas maneras entre sujetos y grupos sociales, sea mediante mecanismos simbólicos y acciones cotidianas, sea como políticas sistemáticas y oficiales de Estados o gobiernos, como en el caso de los regímenes que han aplicado métodos de apartheid (8).
\end{abstract}

Por lo tanto, mientras la zona del ser constituye el privilegio racial, en la zona del no ser se encuentra lo subhumano o no humano; "aquellos que son considerados como diferentes desde el punto de vista de la comunidad ideal o hegemónica, constituida por "nosotros/as" (Weis 2013: 61).

Desafortunadamente, conceptos biologicistas aún surten efecto para jerarquizar las relaciones humanas y justificar las relaciones de poder y de dominación de diversos grupos de personas, tal y como lo hicieron constatar las mujeres participantes, con la pregunta sobre si consideran que la reacción ante una forma de hostigamiento sexual variaría si el hostigador fuese un hombre afrodescendiente o un hombre no racializado (figura 3).

Revista Zona Franca- Centro de estudios interdisciplinario sobre las mujeres (CEIM)- Maestría poder y sociedad desde la problemática de género (MG), Rosario, Argentina. ISSN, 2545-6504 http://zonafranca.unr.edu.ar/index.php/ZonaFrancal Número 29 (2021). 
Figura 3. Consideración de un trato diferenciado entre mujeres

afro y mujeres no racializadas

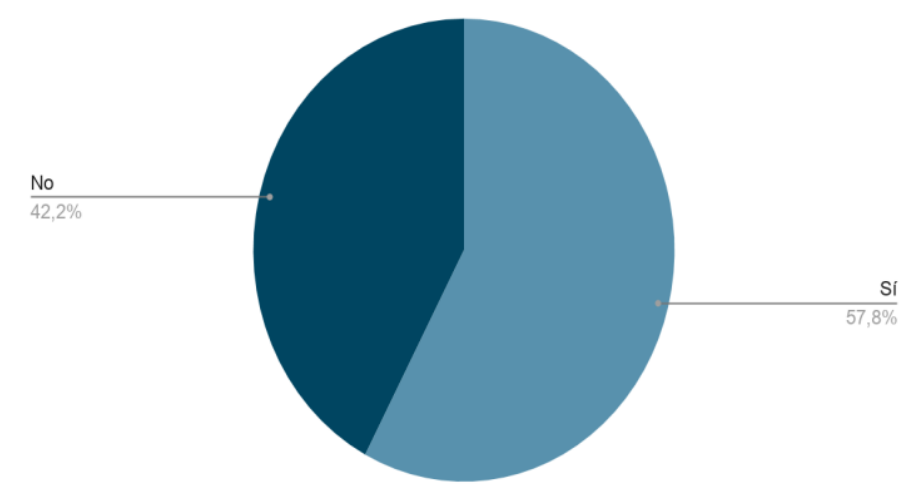

Nota: Elaboración propia.

Mediante esta interrogante, las entrevistadas establecen que existen tratos diferenciados regidos por la etnia. El $88 \%$ de las encuestadas establece que no reaccionaría de forma distinta ante el hostigamiento sexual recibido por parte de hombres afrodescendientes. Ante esto, las mujeres apelan que no debería porqué existir una distinción entre hombres negros y blancos ya que cualquiera que sea el caso debe confirmarse como una expresión violenta meritoria de castigo. Sin embargo, uno de los diecisiete comentarios expuestos señala:

\begin{abstract}
Los hombres afrodescendientes tienen diferente manera de tratar y creo que se considera menos ofensivo el hecho de ser del mismo color de piel, esto se debe a que los hombres no racionalizados tienen a ser más ofensivos en el momento de hostigar y cae más grotesco. $Y$ esto no sólo se remonta al hostigamiento como tal, sino que va más allá de la discriminación que se sigue sufriendo por parte de los demás (mujer, 25 años).
\end{abstract}

La contextualización racial que describe este comentario se considera paralela a uno de los estudios realizados por Nicole Buchannan. Buchannan (2018) evidencia que una de las dificultades que presentan las mujeres afrodescendientes

Revista Zona Franca- Centro de estudios interdisciplinario sobre las mujeres (CEIM)- Maestría poder y sociedad desde la problemática de género (MG), Rosario, Argentina. ISSN, 2545-6504 http://zonafranca.unr.edu.ar/index.php/ZonaFrancal Número 29 (2021). 
al momento de sufrir hostigamiento sexual resulta en el temor de ser acusadas de traición por su propia etnia en el caso que el hostigador sea un hombre negro.

Aunque en esta investigación no se profundiza en la percepción del hostigamiento sexual que sucede específicamente entre personas afrodescendientes, el eje interseccional funciona como análisis significativo para resaltar que las relaciones entre personas se construyen a través de diferentes formas de opresión en las cuales también sucede dominación de unas personas sobre otras.

En consonancia, aunque los sistemas globales de dominación como el patriarcado, el clasismo y el racismo efectivamente operan de manera distinta en mujeres y hombres, "tampoco son sistemas que funcionan de forma separada, sino complejamente interrelacionados" (Cumes, 2009, p.4). Por esto, es esencial abordar el hostigamiento desde un eje integral de atención.

Desde este apartado, se demuestra la importancia de la transversalización de la categoría raza para prevenir y sancionar el hostigamiento sexual en mujeres afrodescendientes. Soslayar las manifestaciones y los efectos de la racialización en la aprehensión del hostigamiento sexual potencia el encubrimiento de las diferentes formas de opresión, las cuales crean desigualdad en todos los espacios de vida de las mujeres y que violenta sus derechos.

\section{Manifestaciones del hostigamiento sexual}

El cuestionario aplicado permite observar y afirmar una serie de manifestaciones del hostigamiento sexual en relación con la documentación estudiada, tales como comentarios, insinuaciones y bromas de carácter sexual. De acuerdo con el apartado anterior, estos comentarios se formulan a partir de elementos raciales y se potencian a través de prejuicios sexuales con respecto de las mujeres negras.

Revista Zona Franca- Centro de estudios interdisciplinario sobre las mujeres (CEIM)- Maestría poder y sociedad desde la problemática de género (MG), Rosario, Argentina. ISSN, 2545-6504 http://zonafranca.unr.edu.ar/index.php/ZonaFranca| Número 29 (2021). 
Sin embargo, la prevalencia de esta forma específica de hostigamiento -según las mujeres encuestadas- carece de identificación por parte de quienes lo sufren, por lo que conlleva a un reconocimiento poco frecuente en sus lugares de trabajo $y$, por ende, a una acción de denuncia reducida.

\section{Percepciones sobre el hostigamiento sexual}

Para este punto del artículo, los aportes de Torres sobre la violencia son esenciales. La autora expone que pensar en la violencia como un acto relacionado con las guerras o con la violencia física podría confirmarse como lo más sencillo. No obstante, las formas en que esta actúa se transforman y varían en contextos determinados, por lo que puede ser tan evidente como un ataque bélico o tan sutil e invisibilizada como la violencia contra las mujeres. Sobre esta última, la autora plantea que solo a través de un buen trabajo de conciencia se podrían identificar manifestaciones de la violencia contra las mujeres como el hostigamiento sexual, la violación o el maltrato de un compañero íntimo.

Lo anterior encuentra su sustento con Segato (2003), quien expone que las diferentes estructuras existentes de violencia se manifiestan como parte de la "normalidad", "lo que sería peor, como un fenómeno "normativo", es decir, que participa del conjunto de las reglas que crean y recrean esa normalidad" (2003: 132). Por lo tanto, los resultados del cuestionario se muestran incongruentes entre la cantidad de mujeres que han percibido comentarios o bromas sexuales respecto de su cuerpo y la poca frecuencia con la que se identifica el hostigamiento sexual en sus lugares de trabajo (Figura 4).

Revista Zona Franca- Centro de estudios interdisciplinario sobre las mujeres (CEIM)- Maestría poder y sociedad desde la problemática de género (MG), Rosario, Argentina. ISSN, 2545-6504 http://zonafranca.unr.edu.ar/index.php/ZonaFranca| Número 29 (2021). 


\section{Figura 4. Frecuencia del hostigamiento sexual}

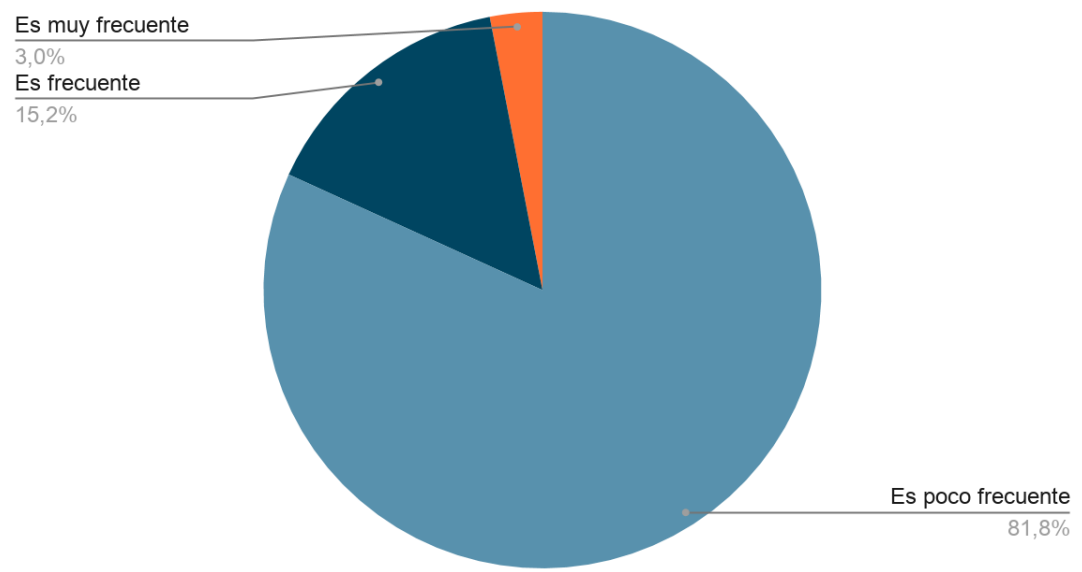

Nota: Elaboración propia.

Con la pregunta "¿Ha recibido comentarios o bromas sexuales respecto de su cuerpo (de manera presencial o por medios tecnológicos o virtuales)?", se desprende que el $78 \%$ de las mujeres participantes afirmó la presencia de estas actitudes en diferentes ámbitos. Sin embargo, dado que estos comportamientos particulares del hostigamiento se combinan con la naturalización simbólica de los estereotipos que se inscriben en los cuerpos, entendemos que la impresión sobre el tema puede sesgarse o advertirse como comportamientos comunes.

\section{Identificación}

En cuanto a la identificación de las personas hostigadoras, los hombres se conforman como los principales victimarios. Lo anterior, y con base en los aportes de Bonino (2002), se sustenta en una génesis patriarcal de las relaciones de género donde la construcción de las masculinidades, principalmente la hegemónica, se apoya en una organización de dominación social y cultural de las relaciones mujer/hombre, principalmente a partir del control y la jerarquización masculina.

A su vez, esta respuesta coincide, igualmente, con encuestas nacionales que muestran que los hombres son, primordialmente, quienes cometen acoso a las

Revista Zona Franca- Centro de estudios interdisciplinario sobre las mujeres (CEIM)- Maestría poder y sociedad desde la problemática de género (MG), Rosario, Argentina. ISSN, 2545-6504 http://zonafranca.unr.edu.ar/index.php/ZonaFranca| Número 29 (2021). 
mujeres. Además, si se retoman los datos arrojados por el Sistema Unificado de Medición de la violencia de Género en Costa Rica (Sumevig, 2018), se puede observar que un total del $97,8 \%$ de las denuncias por hostigamiento suceden en contra de hombres.

Esta conducta, asociada a patrones socioculturales masculinos, se sustenta en un sistema que impone el poder de estos sobre las mujeres, actitud que perjudica seriamente el desenvolvimiento de las mujeres en su espacio laboral. Esto último se correlaciona con la pregunta "¿Cuál es o era la relación laboral con la persona hostigadora?", en la cual cuatro mujeres, de las treinta y tres participantes, manifiesta que el acoso provino de personas en puestos de jefatura.

Es importante rescatar que este dato también coincide con los expuestos por el Sumevig (Sistema Unificado de Medición de la violencia de Género en Costa Rica, 2018), que expone a los compañeros y las compañeras laborales, así como superiores jerárquicos como principales responsables de hostigamiento de acuerdo con la persona denunciante, por lo que se mantiene una perpetración de la erotización del poder sobre quien se considere débil, en este caso, sobre la mujer.

Esta información se correlaciona con la siguiente imagen que expone la relación entre el hostigador con las mujeres encuestadas que recibieron acoso laboral (Figura 6). Cuatro mujeres, de las treinta y tres participantes, manifiesta que el acoso provino de personas en puestos de jefatura. 
Figura 6. Relación laboral con la persona hostigadora

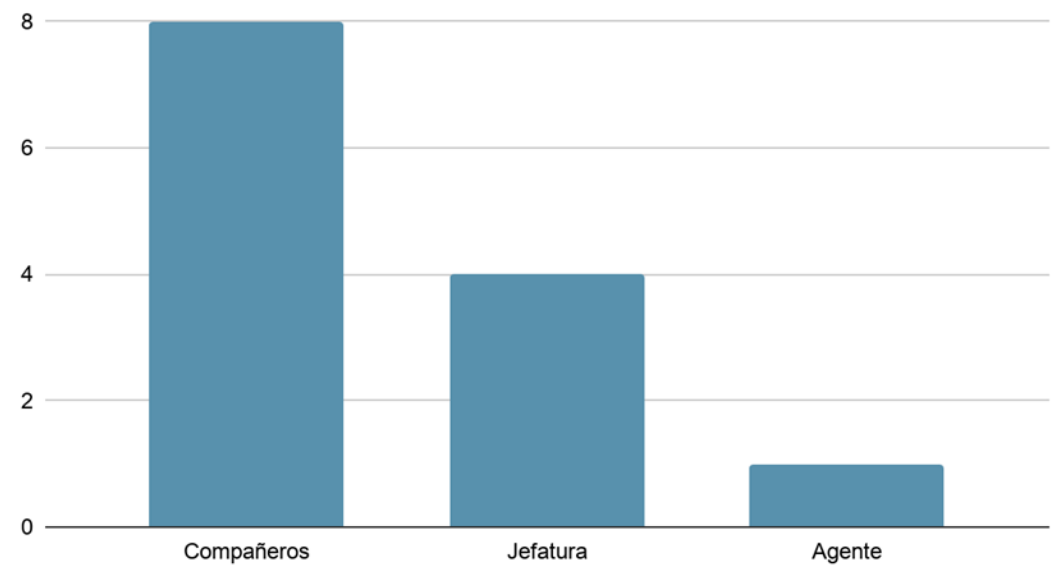

Nota: Elaboración propia.

En la mayoría de respuestas se observa que la relación de compañeros es la más frecuente. Sin embargo, la posición jerárquica de los puestos de trabajo no se explicita, por lo que se vuelve difícil diferenciar si sucedió o no en relaciones de horizontalidad o en relaciones completamente asimétricas, caso diferenciado en cuanto a quienes señalaron la jefatura.

\section{Efectos del hostigamiento}

De acuerdo con la ruta transitada, se evidencia que el hostigamiento sexual es una dinámica social que responde a estructuras patriarcales y racistas que han predominado en todos los espacios ocupados por mujeres.

Sarmiento y Marcelo (2018) advierten de las consecuencias que puede acarrear el hostigamiento sexual, tanto a nivel personal como laboral. Ante esto, se señala que las personas hostigadas pueden desarrollar trastornos a nivel fisiológico y psicológico, con lo que la depresión y el suicidio son las implicaciones más desafortunadas, así como repercusiones laborales que van desde un bajo rendimiento en las actividades hasta la renuncia. 
En consecuencia, las personas que han sufrido hostigamiento sexual pueden presentar tres posibles patrones. El primero, el caso del no reconocimiento del hostigamiento sexual; el segundo, temor a recibir represalias por denunciar la violencia de la cual es víctima y, el tercero, posicionarse como la persona que se atreve a interponer una denuncia. Desafortunadamente, esta última acción parece no llevar a consecuencias positivas. De conformidad con la pregunta “ ¿Ha gestionado alguna denuncia o queja por hostigamiento sexual laboral?", se expone el resultado ante el porcentaje de denuncias, del que siete mujeres de las treinta y tres

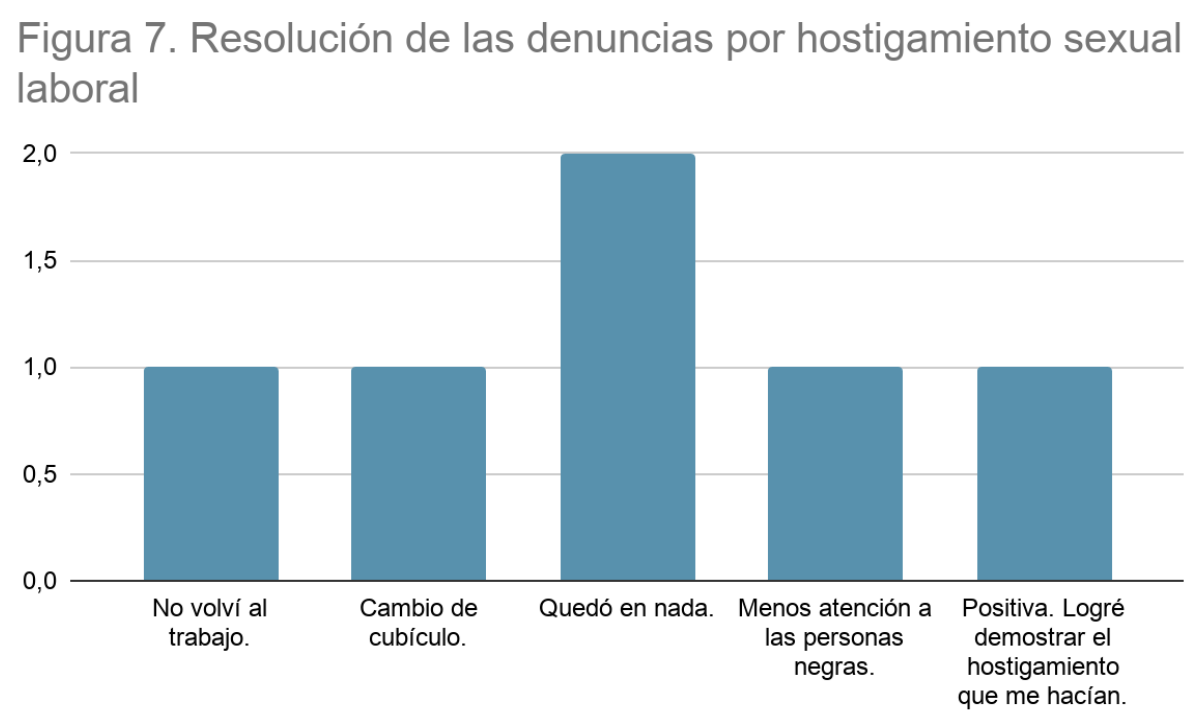

entrevistadas denunciaron actos de hostigamiento (ver figura 7).

Nota: Elaboración propia.

La invisibilización de la violencia contra las mujeres es un patrón sociocultural que permite a los agresores ejercer su poder sin tener repercusiones al respecto; se sostiene un status quo que no se ha podido desestructurar y que reafirma el poder del macho hegemónico sobre las mujeres y sus cuerpos. Muchas veces, el poder de legitimar un cambio se encuentra también en manos de quienes perpetran dicha violencia, por tanto, el avance es lento y el retroceso se afianza. 
De esta forma, es necesario comprender que ante situaciones de acoso puede presentarse el temor a denunciar la violencia sistemática, empezando por la carencia de un sistema que garantice la protección de los derechos de las mujeres $y$, por ende, el acceso a la justicia, por lo que genera que los resultados sean siempre los mismos: "no pasa nada", como mencionan algunas de las mujeres encuestadas.

En la mayoría de los casos estudiados, se evidencian medidas que pueden pensarse como "acciones afirmativas", las cuales afectan la integridad de las mujeres ya que las lleva a tomar decisiones como el abandono de su trabajo o continuar en espacios laborales donde se encuentran cotidianamente frente a su agresor; por ejemplo: "no volví al trabajo" o "me cambiaron de cubículo".

De las siete mujeres que indican la interposición de denuncias, solamente se registra un caso en el que el victimario tuvo repercusiones sobre sus actos. Es importante señalar que la denuncia, aunque se manifieste como inusual, posibilita el fortalecimiento de los sistemas legales y de justicia; igualmente, da voz a las mujeres ante manifestaciones de violencia sexual. La encuestada que realizó la denuncia describe el caso como "positivo, logré demostrar el hostigamiento". Sin embargo, deja entrever que la persona que ha sido hostigada sexualmente debe, en la mayoría de los casos, demostrar que ha sido violentada, con lo que atraviesa por un proceso de revictimización que atenta contra su salud y su dignidad humana.

Finalmente, en respuesta al mismo cuestionamiento, dos mujeres indicaron que la denuncia no tuvo consecuencias. Entendemos con estas respuestas que el acosador quedó absuelto y con esta información verificamos las estadísticas presentadas por el Sumevig (2018), las cuales exteriorizan que este accionar es el más frecuente en el país.

\section{Conclusiones}

Revista Zona Franca- Centro de estudios interdisciplinario sobre las mujeres (CEIM)- Maestría poder y sociedad desde la problemática de género (MG), Rosario, Argentina. ISSN, 2545-6504 http://zonafranca.unr.edu.ar/index.php/ZonaFranca| Número 29 (2021). 
Ante la discusión de los datos arrojados en la encuesta, presentamos algunas reflexiones finales sobre el tema.

Podemos indicar que la discriminación en Costa Rica persiste y con la presente investigación se refleja en el hostigamiento sexual que viven las mujeres afrodescendientes. En su caso se exterioriza con un hostigamiento que se dirige a su cuerpo, a su cultura o a los estereotipos imperantes, lo que implica una violación a sus derechos fundamentales.

Entre las manifestaciones de acoso sexual vivido en el trabajo, estas mujeres reportan, en su mayoría, hostigamiento verbal referido a partes de su cuerpo, con la que entendemos que sus cuerpos se hipersexualizan de acuerdo con unos rasgos físicos que han sido históricamente establecidos en el ideario cultural. En menor medida, se detecta hostigamiento cibernético y no se reportan manifestaciones físicas. Muchas de las frases que exponen como parte del hostigamiento remiten a su trasero, su color de piel o estereotipos asociados con su cultura. Este tipo de expresiones modifican el hostigamiento, el cual no se desprende de la discriminación racial.

La relación raza y género es parte de los ejes interseccionales importantes para evaluar las particularidades de las manifestaciones del hostigamiento sexual. Consideramos pertinente, en futuras investigaciones, profundizar en los aspectos que transversalizan a las mujeres para continuar con el diálogo y la erradicación de esta problemática.

La pertenencia al mismo grupo étnico convierte la violencia sexual (hostigamiento sexual) de los hombres afro costarricenses, en acciones válidas y justificables. En este caso, el hostigamiento que no incorpora elementos asociados a su etnicidad no siempre es considerada un abuso de poder.

Las mujeres afrodescendientes que han percibido tratos diferentes con respecto a las mujeres no racializadas se distribuyen a lo largo del país. Las

Revista Zona Franca- Centro de estudios interdisciplinario sobre las mujeres (CEIM)- Maestría poder y sociedad desde la problemática de género (MG), Rosario, Argentina. ISSN, 2545-6504 http://zonafranca.unr.edu.ar/index.php/ZonaFrancal Número 29 (2021). 
respuestas obtenidas provienen de diferentes provincias: Limón, Guanacaste, Heredia, San José, Cartago; lo cual implica no solamente la diversidad étnica sino la distribución de discriminación en el país.

El hostigamiento sigue siendo naturalizado por algunas mujeres que participaron en la encuesta, por lo que la identificación del problema se dificulta y el encubrimiento es lo común. Ante este inconveniente, resaltamos la necesidad de concienciar a la población sobre las conductas que implican hostigamiento, la necesidad de educar sobre las medidas que se deben tomar para protegerse, así como las instancias en las que se debe denunciar.

Por otro lado, la denuncia en los casos expuestos en la encuesta manifiesta problemáticas como la ausencia de sanciones para el acosador o la incapacidad de resarcir a la víctima. Aun así, se evidencia en dos casos que el haber gestionado la denuncia deja en las víctimas la sensación de señalar al culpable de un crimen y socializar un acto dañino, a pesar de la revictimización que pueden vivir.

Este llamado a la conciencia y a la educación sobre el tema debe darse, idealmente, a nivel general dentro de la sociedad, dado que la denuncia debe implicar protección a la víctima ante cualquier circunstancia de parte de las jerarquías institucionales. En algunos casos, las entrevistadas no denuncian por temor al escarnio público y la revictimización o repercusiones en su puesto de trabajo, a pesar de que se deberían sentir protegidas y apoyadas en circunstancias relacionadas con esta problemática.

Aunado a lo anterior, se confirma que el hostigamiento sexual vulnerabiliza a las mujeres y genera en ellas consecuencias devastadoras, como la pérdida de trabajo reportado por una de las mujeres entrevistadas.

En la encuesta, los hombres son identificados como los principales hostigadores, por lo que entendemos que estas conductas se asocian, indiscutiblemente, con un sistema social y cultural que ha oprimido a las mujeres y

Revista Zona Franca- Centro de estudios interdisciplinario sobre las mujeres (CEIM)- Maestría poder y sociedad desde la problemática de género (MG), Rosario, Argentina. ISSN, 2545-6504 http://zonafranca.unr.edu.ar/index.php/ZonaFranca| Número 29 (2021). 
ha establecido que los hombres dominen los estratos públicos al punto que se normalizan las conductas hostiles hacia las mujeres. Consideramos, finalmente, que a lo largo de las últimas décadas se han dado avances sustanciales en materia de derechos humanos en Costa Rica en el que se busca la inclusión de las personas afrocostarricenses, como la modificación del artículo 1 de la Constitución en 2015, en el que se indica que no solo es una nación libre e independiente sino también multiétnica y pluricultural, así como la Política Nacional para una Sociedad Libre de Racismo, Discriminación y Xenofobia (2014-2025)

Aun así, es indispensable establecer cambios sustanciales en las poblaciones afrocostarricenses, como la toma de conciencia sobre los tratos discriminatorios y su reacción ante ellos a partir de la crítica y la denuncia. Para ello debe darse un cambio respecto de la construcción de masculinidades hegemónicas ligadas a patrones culturales heterosexistas y racistas, los cuales llevan a la justificación de conductas degradantes hacia las mujeres. Como propone Connell, la masculinidad no es un hecho empírico, expuesto así por el psicoanálisis clásico, ni un arquetipo eterno como lo proponía Jung, se trata de algo que ocupa un lugar simbólico y está atado a cuestiones políticas, por lo que existe la posibilidad de rechazar todo lo que esta conlleva: "la masculinidad y la feminidad se entienden fácilmente como roles sexuales internalizados, productos del aprendizaje social o socialización" (Conell 2003: 37 y 41). De este modo, desde la infancia, tanto mujeres como hombres, deben recibir una formación basada en el principio de la igualdad y que se transmita a partir de procesos de socialización construidos con proyecciones en un futuro justo y equitativo, con el fin de transformar las masculinidades y las percepciones raciales.

\section{Bibliografía}

AYALA, Jonathan, CABEZAS, Andrés y FILIPPIS, Gala (2011). "La integración de la mujer en el ámbito laboral". Revista Poiésis, № 22, 1-11. https://www.funlam.edu.co/revistas/index.php/poiesis/article/view/212

Revista Zona Franca- Centro de estudios interdisciplinario sobre las mujeres (CEIM)- Maestría poder y sociedad desde la problemática de género (MG), Rosario, Argentina. ISSN, 2545-6504 http://zonafranca.unr.edu.ar/index.php/ZonaFranca| Número 29 (2021). 
BONINO, Luis (2002). "Masculinidad hegemónica e identidad masculina". $\begin{array}{llllll}\text { Dossiers } & \text { Feministes } & \text { 6: } & \text { Mites, } & \text { № } & \text { 6, }\end{array}$ https://www.raco.cat/index.php/DossiersFeministes/article/viewFile/102434/153629

BOURDIEU, Pierre. (1998) La dominación masculina. Barcelona: Editorial Anagrama.

CARVAJAL, Zaira y DELVÓ, Patricia (2010) "Análisis comparativo de dos estudios sobre hostigamiento sexual en la población estudiantil de la Universidad Nacional”. Revista Latinoamericana de Derechos Humanos, №2, 198-214. Recuperado de: http://www.flacsochile.org/publicaciones/nuevos-desafios-para-lainclusion-social-y-la-equidad-en-la-educacion-superior/

CONNELL, Raewyn (2003). Masculinidades. México: Universidad Nacional Autónoma de México.

CRENSHAW, Kimberlé (1995). "The Identity Factor in Multiculturalism". Liberal Education, № 4, 6-11. Recuperado de: EBSCOhost, search.ebscohost.com/login.aspx?direct=true \&db=ehh\&AN=9512131400\&lang=es $\underline{\text { \&ite }=\text { ehost-live \&scope }=\text { site }}$

CUMES, Aura (2009). "Multiculturalismo, género y feminismos: mujeres diversas, luchas complejas". En Pequeño, A. (comp.) Participación y políticas de mujeres indígenas en contextos latinoamericanos recientes. Quito: FLACSO/Ministerio de Cultura del Ecuador. Recuperado de: https://biblio.flacsoandes.edu.ec/libros/digital/41463.pdf

FOUCAULT, Michel. (1992). Historia de la sexualidad. Madrid: Siglo Veintiuno editores, S.A. Tomo I.

HERNÁNDEZ Roberto, FERNÁNDEZ, Carlos y BAPTISTA, María del Pilar (2010). Metodología de la investigación. Quinta edición. Editorial, Mc. Graw Hill, México.

HOLMES, Tamara (2018). "Sexual Harassment on the Job“. Essence, № 49, 77-80.

Recuperado

de:

http://una.remotexs.co/user/login?dest=http://search.ebscohost.com.una.remotexs.

Revista Zona Franca- Centro de estudios interdisciplinario sobre las mujeres (CEIM)- Maestría poder y sociedad desde la problemática de género (MG), Rosario, Argentina. ISSN, 2545-6504 http://zonafranca.unr.edu.ar/index.php/ZonaFranca| Número 29 (2021). 
$\underline{\text { co/login.aspx?direct=true\&AuthType }=i p, u i d, u r l \& d b=a s n \& A N=129416575 \& l a n g=e s}$

\&site=ehost-live

HOPENHAYN, Martín y BELLO, Álvaro (2001). "Discriminación étnico-racial y xenofobia en América Latina y el Caribe", Serie Políticas Sociales, № 47 (LC/L.1546-P). Santiago de Chile: CEPAL. Recuperado de: https://repositorio.cepal.org/bitstream/handle/11362/5987/1/S01050412 es.pdf

Inamu (2016). Acoso sexual en el trabajo. INAMU, San José, Costa Rica. Recuperado de: https://www.inamu.go.cr/acoso-sexual-en-el-trabajo

Inamu (2017). La Política Nacional para la Atención y la Prevención de la Violencia contra las Mujeres 2017-2032. Recuperado de: https://www.inamu.go.cr/documents/10179/967660/PLANOVI+20172032/fcb84a5b-cb73-4ce0-a08c-a1b886180b02

Inamu (2019). Tercer Estado de los Derechos Humanos de las Mujeres en Costa Rica. San José: Instituto Nacional de las Mujeres. Recuperado de: https://www.inamu.go.cr/documents/10179/275546/Tercer+Estado+de+los+Derech os+Humanos+de+las+Mujeres+en+Costa+Rica+\%282019\%29.pdf/f1affa72-985f4489-bcf6-28206a661e4d

Inamu. Mujeres Afrodescendientes proponen ruta para alcanzar igualdad de derechos. Comunicación. 2021. Recuperado de https://www.inamu.go.cr/mujeresafro

Ley de modificación de la Ley contra el hostigamiento sexual en el empleo y la docencia ( $\mathrm{N}^{\circ}$ 8805, 2011) Costa Rica. Recuperado de: https://www.cso.go.cr/legislacion/leyes/ley n 7476 contra hostigamiento sexual. $\underline{\mathrm{pdf}}$

LUGONES, María (2012). "Subjetividad esclava, colonialidad de género, marginalidad y opresiones múltiples". En Patricia Montes (Editora), Pensando los feminismos en Bolivia (129-140). La Paz: La Creativa. Recuperado de: http://catolicasbolivia.org/wp-content/uploads/2017/09/trab.-pensando-los$\underline{\text { feminismos-en-bolivia.pdf\#page }=129}$

Revista Zona Franca- Centro de estudios interdisciplinario sobre las mujeres (CEIM)- Maestría poder y sociedad desde la problemática de género (MG), Rosario, Argentina. ISSN, 2545-6504 http://zonafranca.unr.edu.ar/index.php/ZonaFrancal Número 29 (2021). 
MIGNOLO, Walter (2009) "La colonialidad a lo largo y ancho: el hemisferio occidental en el horizonte colonial de la modernidad". Lander, E. (comp.), La colonialidad del saber: eurocentrismo y ciencias sociales. Buenos Aires: CLACSO. Recuperado de: http://biblioteca.clacso.edu.ar/clacso/sursur/20100708044529/5 mignolo.pdf

OIT (Organización Internacional de Trabajo) (2013). Hoja informativa sobre el hostigamiento o acoso sexual. América Central, Haití, Panamá y República Dominicana: Equipo Técnico de Trabajo Decente de la OIT. Recuperado de: https://www.ilo.org/sanjose/publicaciones/WCMS 227404/lang--es/index.htm

RAMÍREZ (2016). Protocolo para la Detección y Denuncia del Hostigamiento Sexual en la Universidad Nacional. Heredia: Programa de publicaciones e impresiones de la Universidad Nacional.

REYES, Noilly (2008). "La falsedad de la universalidad: El hostigamiento sexual como una violación de los derechos de las mujeres". Revista de Derechos Humanos, № 9, 126-139. Recuperado de https://repositorio.una.ac.cr/bitstream/handle/11056/7889/lafalsedaddelauniversalid a Nreyes.pdf?sequence $=1$

SAGOT, Monserrat (2008) "Los límites de las reformas: Violencia contra las mujeres y Políticas Públicas en América Latina". Revista de Ciencias Sociales, № 120, 35-48. Recuperado de: https://www.redalyc.org/pdf/153/15312721004.pdf

SÁNCHEZ-ARTEAGA, Juan Manuel, SEPÚLVEDA, Claudia y EL-HANI, Charbel (2013). "Racismo científico, procesos de alterización y enseñanza de ciencias". Revista Internacional de Investigación en Educación, № 12, 55-67. Recuperado de: https://revistas.javeriana.edu.co/index.php/MAGIS/article/view/7201

SARMIENTO, Patricia y Marcelo, Yuri (2018). Aproximación de la problemática del hostigamiento sexual laboral contra las mujeres: supervisión a ministerios, gobiernos regionales y poder judicial. Lima: Defensoría del pueblo.

SEGATO, Rita (2003). "Las estructuras elementales de la violencia: contrato y status en la etiología de la violencia”. En Garzón Baltazar (Editor), Las estructuras

Revista Zona Franca- Centro de estudios interdisciplinario sobre las mujeres (CEIM)- Maestría poder y sociedad desde la problemática de género (MG), Rosario, Argentina. ISSN, 2545-6504 http://zonafranca.unr.edu.ar/index.php/ZonaFranca| Número 29 (2021). Página 322 
elementales de la violencia (131-148). Brasilia: Universidad Nacional de Quilmes Editorial.

SUMEVIG (Sistema Unificado de Medición de la violencia de Género en Costa Rica). (2018) Indicadores 2012- 2016, Costa Rica: Inec. Recuperado de: https://www.inamu.go.cr/sumevig

TORRES, Marta. "Violencia social y violencia de género". Recuperado de: Planovicr.org. S.f. Recuperado de: americalatinagenera.org/newsite/.../doc 2 Violencia-social-y-violencia-degénero.do

WADE, Peter; Urrea, Giraldo y Viveros Vigoya Mara (Eds.) (2008). En Raza, etnicidad y sexualidades. Bogotá: Universidad Nacional de Colombia. 